\title{
Kai-Xin-San, a traditional Chinese medicine formulation, exerts antidepressive and neuroprotective effects by promoting pCREB upstream pathways
}

\author{
XIAN-ZHE DONG ${ }^{1}$, DONG-XIAO WANG ${ }^{1}$, BING-YING YU $^{2}$, PING LIU $^{1}$ and YUAN HU ${ }^{1}$ \\ ${ }^{1}$ Department of Clinical Pharmacology, General Hospital of Chinese People's Liberation Army, Beijing 100853; \\ ${ }^{2}$ Department of Pharmacy, Hebei North University, Zhangjiakou, Hebei 075000, P.R. China
}

Received July 28, 2015; Accepted September 15, 2016

DOI: $10.3892 / \mathrm{etm} .2016 .3773$

\begin{abstract}
Kai-Xin-San (KXS) is a traditional Chinese medicine that has been widely used for the treatment of emotion-related disease. However, the underlying mechanism remains largely unknown. The present study aimed to examine whether phospho-cAMP response element-binding protein (pCREB) and upstream components, such as extracellular signal-regulated kinase (ERK), phospho-ERK (pERK), phosphatidylinositol-3-kinase (PI3K), protein kinase B (Akt), glycogen synthase kinase $3 \beta(\mathrm{GSK} 3 \beta)$ and pGSK3 $\beta$ are associated with the antidepressive effect of KXS. In total, 24 male Wistar rats were randomly divided into three groups, including control ( $\mathrm{n}=8$, no treatment), induced with chronic unpredictable mild stress (CMS) $(n=8)$, and CMS rats treated with KXS at dosage of $370 \mathrm{mg} / \mathrm{kg} /$ day orally. Primary hippocampal neuronal cultures were prepared from Wistar rats for cell survival and proliferation assays. In KXS rats, increased
\end{abstract}

Correspondence to: Professor Ping Liu or Professor Yuan Hu, Department of Clinical Pharmacology, General Hospital of Chinese People's Liberation Army, 28 Fuxing Road, Beijing 100853, P.R. China

E-mail: cpi301@163.com

E-mail: huyuan1980619@126.com

Abbreviations: KXS, Kai-Xin-San; BDNF, brain-derived neurotrophic factor; CREB, cAMP response element-binding; p-CREB, phospho-cAMP response element-binding protein; CMS, chronic mild stress; TrkB, receptor tyrosine kinase B; ERK, extracellular signal-regulated kinase; pERK, phospho-extracellular signal-regulated kinase; PI3K, phosphatidylinositol-3-kinase; Akt, protein kinase $\mathrm{B}(\mathrm{PKB})$; GSK3 $\beta$, glycogen synthase kinase $3 \beta$; pGSK3 $\beta$, phospho-glycogen synthase kinase $3 \beta$; DMSO, dimethyl sulfoxide; MTT, 3-(4,5-Dimethylthiazol-2-yl)-2,5-diphenyltetrazolium bromide; OD, optical density; PBS, phosphate-buffered saline; NGF, nerve growth factor; MAP-2, microtubule-associated protein 2; DAPI, 4',6-diamidino-2-phenylindole

Key words: antidepressant effect, Kai-Xin-San, brain-derived neurotrophic factor, extracellular signal-regulated kinase signal pathway, traditional Chinese medicinal formula protein expression levels of pCREB, BDNF and tyrosine receptor kinase $\mathrm{B}$ (TrkB) were observed in the hippocampus and prefrontal cortex, compared with the CMS model group. Furthermore, increased expression levels of ERK, pERK, PI3K, Akt, and GSK3 $\beta$ were also detected in the hippocampus and prefrontal cortex of KXS-treated rats compared with CMS model rats and in primary hippocampal neuronal cells treated with KXS. These results suggest that pCREB and upstream components, including TrkB/ERK/CREB and TrkB/PI3 K/CREB, may contribute to the antidepressive effect induced by KXS. Further studies are required to confirm these findings.

\section{Introduction}

It has previously been demonstrated that brain-derived neurotrophic factor (BDNF), which is a neurotrophic factor, has a key role in depression. In depressive patients with serum reduced levels of BDNF, BNDF increased after treatment with antidepressant agents $(1,2)$. A postmortem study reported a significant decrease in BDNF levels in the hippocampi of depression-induced suicide subjects, but not in suicide subjects treated with antidepressants (3). Further studies have demonstrated that downstream components of the BDNF/tyrosine receptor kinase B (TrkB) signaling pathway, such as the phosphatidylinositol-3-kinase $(\mathrm{PI} 3 \mathrm{~K}) /$ phosphatidylinositol-3-kinase (Akt) pathway, were inhibited in depressive patients (3-6). The phospho-cAMP response element-binding protein ( $\mathrm{pCREB}$ ) may regulate the transcription of downstream genes, including BDNF, potentially by binding to response elements in the promoter regions of gene transcription, thus regulating neurotrophy, differentiation, cell viability and recognition functions (7-10).

Kai-Xin-San (KXS) is a well-known traditional Chinese medicine first recorded in Bei Ji Qian Jin Yao Fang (Thousand Formulae for Emergency Thousand Formulae for Emergency) by Sun Si-Miao $(11,12)$. KXS consists of ginseng (Panax ginseng C.A. Meyer), hoelen (Poria cocos F.A. Wolf), polygala (Polygala tenaifolia Willd) and acorus (Acorus tatarinowii Schott) at the ratio of 3:3:2:2 (13). It has been widely used in the treatment of depression, as well as in learning and memory deficits for thousands years (14). In our previous studies, we 
have demonstrated the anti-depressive effect of KXS in rats by utilizing chronic unpredictable mild stress (CMS), the tail suspension test, the forced swim test and chronic fatigue syndrome; in addition, increased levels of BDNF and pCREB were detected, suggesting that they may have roles in the anti-depressive effect of KXS (15-18). The present study aimed to explore the mechanism of KXS via the regulation of the $\mathrm{BDNF}$ and pCREB pathways in rats and neuronal cells.

\section{Materials and methods}

KXS preparation. Herbs for KXS (ginseng, hoelen, polygala, and acorus) were purchased from LvYe Medicinal Material Company (Beijing, China), and the quality met the requirements outlined in the Chinese Pharmacopoeia (2010) (19). The voucher specimen was registered and deposited in the Herbarium of the Traditional Chinese Medicinal pharmacy. KXS was supplied in powder form derived from a mixture of the aqueous extract as described previously (20). Briefly, $1.5 \mathrm{~kg}$ ginseng, $1.5 \mathrm{~kg}$ hoelen, $1 \mathrm{~kg}$ polygala and $1 \mathrm{~kg}$ acorus gramineus was soaked in 501 water for $3 \mathrm{~h}$ and was subsequently extracted three times using a circumfluence extraction method. The aqueous extracts were filtered and evaporated under reduced pressure. The obtained concentrates were freeze-dried to powder form and stored at $4^{\circ} \mathrm{C}$. Average yield was $1 \mathrm{~g} \mathrm{KXS}$ powder/4.83 g total herbs. KXS powder was standardized using a high-performance liquid chromatography-fingerprint method, as previously described (21).

Animals. In total, 24 male Wistar rats, weighing $180 \pm 10 \mathrm{~g}$ at 6 weeks, were obtained from the Animal Breeding Center of the People's Liberation Army general hospital (Beijing, China). All rats were housed in a temperature- $\left(23 \pm 2{ }^{\circ} \mathrm{C}\right)$ and humidity-controlled $(60 \pm 10 \%)$ facility with a 12 -h light/dark cycle with free access to food and water. All animal experimental protocols were approved by the Animal Experimentation Ethics Committee of General Hospital of Chinese PLA (X5-2013-04). All animal handling procedures were performed in accordance with the Principles of Laboratory Animal Care and Chinese legislation for the use and care of laboratory animals.

Induction of CMS. CMS was induced in 16 rats following the established protocol (22). Rats received 4 weeks of stress stimulation, which included high-speed agitation (10 min), deprivation of food or water ( $24 \mathrm{~h})$, immobilization $(2 \mathrm{~h})$, continuous illumination $(24 \mathrm{~h})$, tilted cage $(12 \mathrm{~h})$ and forced swimming in ice water (5 min). Rats were randomly assigned one stimulation daily at 3-5 p.m. for four weeks. The remaining eight rats were housed undisturbed without contact with the stressed animals as the control group.

Drug administration. The 16CMS-induced rats were randomly divided into two groups treated with either water ( $n=8$; CMS group) or KXS at $370 \mathrm{mg} / \mathrm{kg}$ orally at 9-10 am daily for three weeks (n=8; KXS group).

Sucrose-preference test. Sucrose-preference test was performed to define anhedonia prior to surgery (baseline) and after CMS induction. Rats were food-and water-deprived for $18 \mathrm{~h}$, and subsequently fed with pre-weighted bottles containing $1 \%$ sucrose solution and water for $1 \mathrm{~h}$. Intake was measured by weighing the bottles before and after each test. All tests were carried out in the home cage to minimize extraneous novelty and disturbance. Sucrose preference was calculated as: Sucrose preference $=$ sucrose intake $/$ (sucrose intake + water intake). Anhedonia was defined as a reduction in sucrose preference relative to baseline levels.

Open-field test. Locomotor activity was assessed to detect immobility or changes in motor activity in an open-field test. A metallic cubic open field arena $(80 \times 80 \times 80 \mathrm{~cm})$ was used. The floor of the box was 25 squares (5 squares long; 5 squares wide). Rats were individually placed into the center of the arena and allowed to explore freely for $3 \mathrm{~min}$. The floor of the open-field apparatus was wiped cleaned with $70 \%$ ethanol between tests. The behavior, including the number of square lines crossed with all four paws, and rearing, including the number of times each rat stood on its hind limbs, were recorded.

Western blot. At the end of experiment, all rats were sacrificed with $10 \%$ chloral hydrate solution $(3.5 \mathrm{ml} / \mathrm{kg}$; i.p.). Hippocampus and prefrontal cortex tissues were collected and lysed with radioimmunoprecipitation assay buffer (Applygen Technologies, Inc., Beijing, China), schizolysised for $20 \mathrm{~min}$ on ice and centrifuged at $12,000 \times \mathrm{g}$ for $10 \mathrm{~min}$ at $4^{\circ} \mathrm{C}$. Protein samples were heated at $95^{\circ} \mathrm{C}$ for $8 \mathrm{~min}$, separated by $12 \%$ SDS-PAGE and transferred to nitrocellulose membranes. Membrane were blocked for $2 \mathrm{~h}$ in TBST (25 mM Tris, $140 \mathrm{mM}$ $\mathrm{NaCl}, 27 \mathrm{mM} \mathrm{KCl}$ and $0.02 \%$ Tween 20 ) containing $5 \%$ bovine serum albumin and incubated with primary antibodies specific for BDNF (ab108319, 1:200)(Abcam, Cambridge, MA, USA), TrkB (BS1431, 1:500), ERK (AP0491, 1:500), pERK (BS4621, 1:500), Akt (BS1502, 1:500), PI3K (BS3678, 1:500), pGSK3 $\beta$ (BS4084, 1:500) and GSK3 $\beta$ (BS1402, 1:500; Bioworld Technology, Inc., St. Louis Park, MN, USA), pCREB (\#9198, 1:1,000) and CREB (\#9197, 1:1,000; Cell Signaling Technology, Inc., Danvers, MA, USA) at $4^{\circ} \mathrm{C}$ over night. Following three washes with TBST, membranes were incubated for $1 \mathrm{~h}$ at room temperature with horseradish peroxidase-labeled secondary antibodies (BS13278, 1:5,000; Bioworld Technology, Inc.), washed with TBST three times. Blots were developed using a electrochemiluminescence system (UVP LLC, Upland, CA, USA).

Primary hippocampal neurons culture. Primary hippocampal neuronal cultures were prepared as described previously (23), with a few modifications. A total of 36 Newborn Wistar rats (age, $<24 \mathrm{~h}$ ) were purchased from the Peking University Health Science Center (Beijing, China). Hippocampi were dissected on ice, minced and digested with $0.25 \%$ trypsin for $15 \mathrm{~min}$ at $37^{\circ} \mathrm{C}$. Susequently, the samples were supplemented with $5 \mathrm{ml}$ fetal bovine serum (FBS) to terminate the digestion procedure. Cell suspension was passed through a 200 mesh (diameter, $74 \mu \mathrm{m}$ ) cell strainer and separated by density gradient centrifugation for $20 \mathrm{~min}$ at $300 \mathrm{x} g$ at room temperature. The suspension was centrifuged for $5 \mathrm{~min}$ at $225 \mathrm{x} \mathrm{g}$ at room temperature and the pellet was resuspended in $10 \mathrm{ml}$ Dulbecco's modified Eagle's medium (DMEM). Following the measurement of cell density using a hemacytometer, the 
Table I. Effects of KXS on behavioral indices in the CMS-treated rats (n=8/group).

\begin{tabular}{lcccc}
\hline Group & Lines crossed $(\mathrm{n})$ & Rearing count $(\mathrm{n})$ & Sucrose intake $(\mathrm{ml})$ & Body weight $(\mathrm{g})$ \\
\hline Control & $68.9 \pm 8.5$ & $9.0 \pm 2.1$ & $90.54 \pm 3.98$ & $422.3 \pm 24.4$ \\
CMS & $15.4 \pm 6.2^{\mathrm{a}}$ & $3.8 \pm 2.0^{\mathrm{a}}$ & $70.94 \pm 7.09^{\mathrm{a}}$ & $352.4 \pm 31.1^{\mathrm{a}}$ \\
KXS & $36.8 \pm 8.6^{\mathrm{c}}$ & $6.6 \pm 2.5^{\mathrm{b}}$ & $91.16 \pm 8.49^{\mathrm{b}}$ & $388.3 \pm 17.4^{\mathrm{b}}$ \\
\hline
\end{tabular}

CMS rats were exposed to stressors for four weeks. Following CMS exposure, KXS group rats were treated with $370 \mathrm{mg} / \mathrm{kg} / \mathrm{day} \mathrm{KXS} \mathrm{for} \mathrm{three}$ weeks. Data are presented as the mean \pm standard deviation. ${ }^{\mathrm{a}} \mathrm{P}<0.01 \mathrm{vs}$. the control group; ${ }^{\mathrm{b}} \mathrm{P}<0.05$ and ${ }^{\mathrm{c}} \mathrm{P}<0.01 \mathrm{vs}$. the model group. CMS, chronic unpredictable mild stress; KXS, Kai-Xin-San.
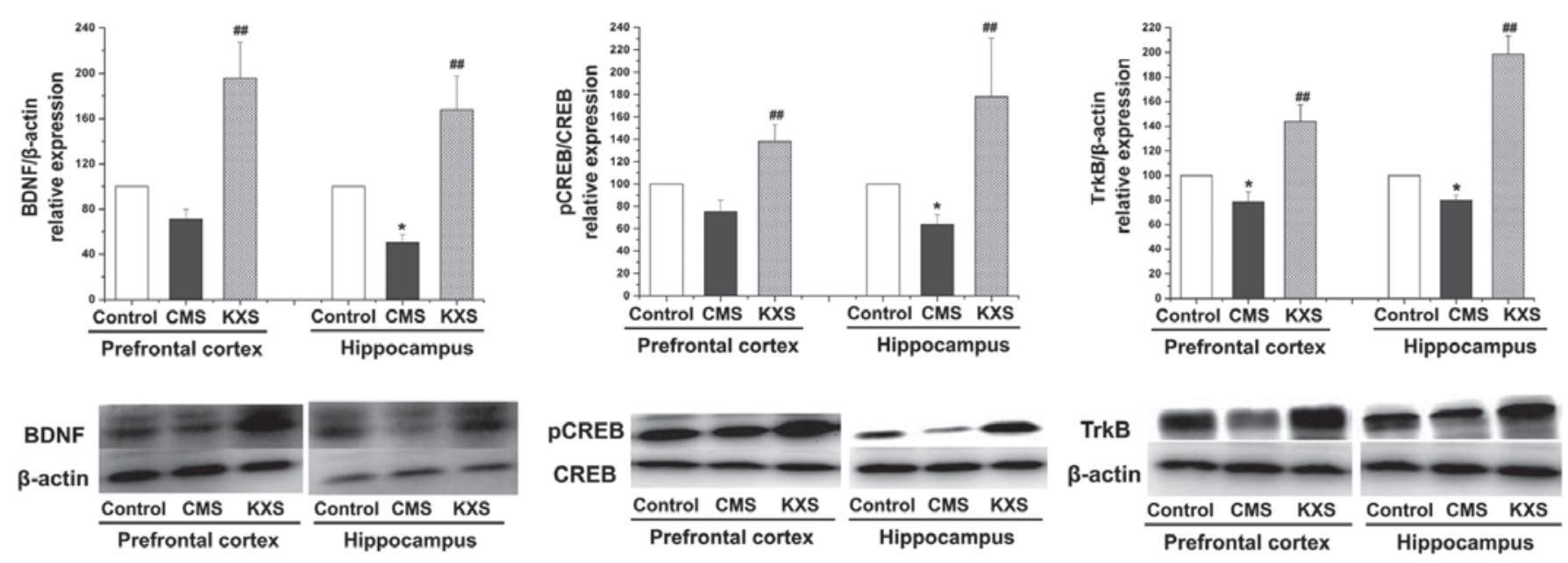

Figure 1. Effects of KXS on the TrkB/BDNF signaling pathway in the control and CMS rats. KXS (370 mg $/ \mathrm{kg})$ was given daily for three weeks. Data are presented as the mean \pm standard deviation. ${ }^{*} \mathrm{P}<0.05$ vs. the control group, ${ }^{\# \#} \mathrm{P}<0.01$ vs. the model group. CMS, chronic unpredictable mild stress; KXS, Kai-Xin-San; BDNF, brain-derived neurotrophic factor; p, phospho; CREB,cAMP response element-binding protein; TrkB, tyrosine receptor kinase B.

neuronal cells were plated into 6 -well plates at $5 \times 10^{5} \mathrm{cell} / \mathrm{ml}$ for culture in DMEM supplemented with $10 \% \mathrm{FBS}, 1 \mathrm{mmol} / \mathrm{l}$ glutamine, $20 \mathrm{mmol} / 1$ sodium pyruvate, and $10 \mathrm{ng} / \mathrm{ml}$ nerve growth factor (Gibco; Thermo Fisher Scientific, Inc., Waltham, MA, USA). 200 nM K252a (Sigma Aldrich; Merck Millipore, Darmstadt, Germany) was added to cells $30 \mathrm{~min}$ before the exposure to KXS as an inhibitor of TrkB. Cells were used for assay analysis at day 7 when the percentage was $\sim 85 \%$, as assessed by microtubule-associated protein 2 (MAP-2) immunostaining.

Cell survival and proliferation assays. Cell viability was evaluated via MTT assay (24). Briefly, $20 \mu 1 \mathrm{MTT}$ solution $(2 \mathrm{mg} / \mathrm{ml}$ in PBS) was added at a final concentration of $0.5 \mathrm{mg} / \mathrm{ml}$ and incubated at $37^{\circ} \mathrm{C}$ for $4 \mathrm{~h}$. Following removal of the medium, $150 \mu 1$ DMSO was added. Optical densities were subsequently read using a microplate reader at $570 \mathrm{~nm}$ (1420 Vitor 3; Perkin-Elmer, Waltham, MA, USA). Viability was expressed as a percentage of the KXS-treated cells according to the control cells.

Statistical analysis. All data were expressed as the mean \pm standard error. Differences between groups were analyzed by one-way analysis of variance followed by Dunnett's test. Statistically analyses were performed using SPSS 17.0 software (SPSS, Inc., Chicago, IL, USA). P<0.05 was considered to indicate a statistically significant difference.

\section{Results}

Behavioral index. Table I shows the results from the open-field and sucrose-preference tests. The index of sucrose preference in the CMS group was significantly lower than that of the control group $(\mathrm{P}<0.01)$ and the KXS group $(\mathrm{P}<0.05)$. Similarly, the number of lines crossed and rearing values in the CMS group were significantly lower than that of the control group $(\mathrm{P}<0.01)$ and the KXS group $(\mathrm{P}<0.01, \mathrm{P}<0.05)$, suggested that KXS could improve the depressive symptom in rats induced by CMS.

Protein expression levels of BDNF, CREB and TrkB. Western blot analysis demonstrated that the protein expression levels of BDNF, pCREB and TrkB in the hippocampus and TrkB in the prefrontal cortex were significantly reduced in the CMS group compared with the control group $(\mathrm{P}<0.05)$. Furthermore, the protein expression levels of BDNF, pCREB and TrkB in the hippocampus and prefrontal cortex were increased in the KXS group compared with the CMS group $(\mathrm{P}<0.01$; Fig. 1$)$, suggested that the antidepressive effect of KXS might be involved in its regulating on BDNF and TrkB through promoting the $\mathrm{pCREB}$ level. 

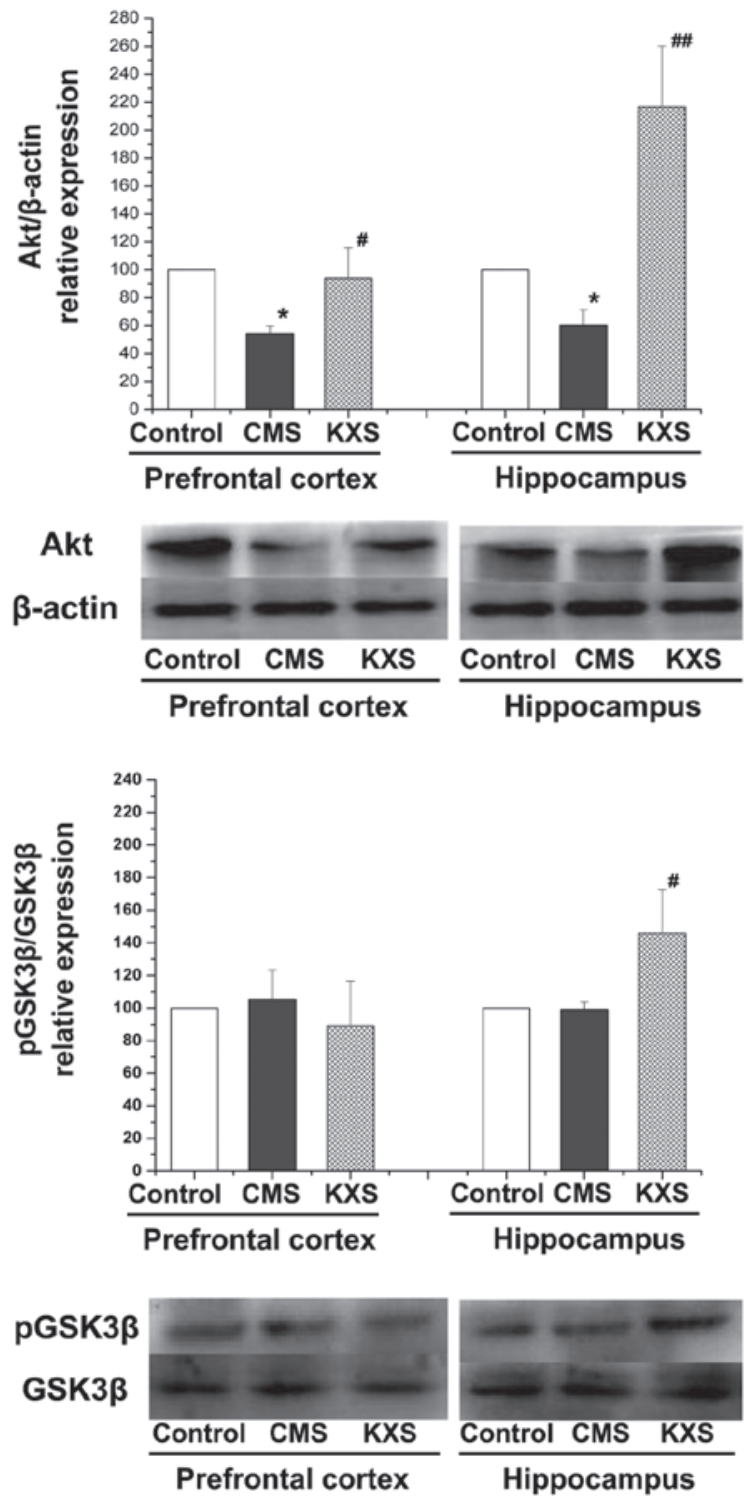
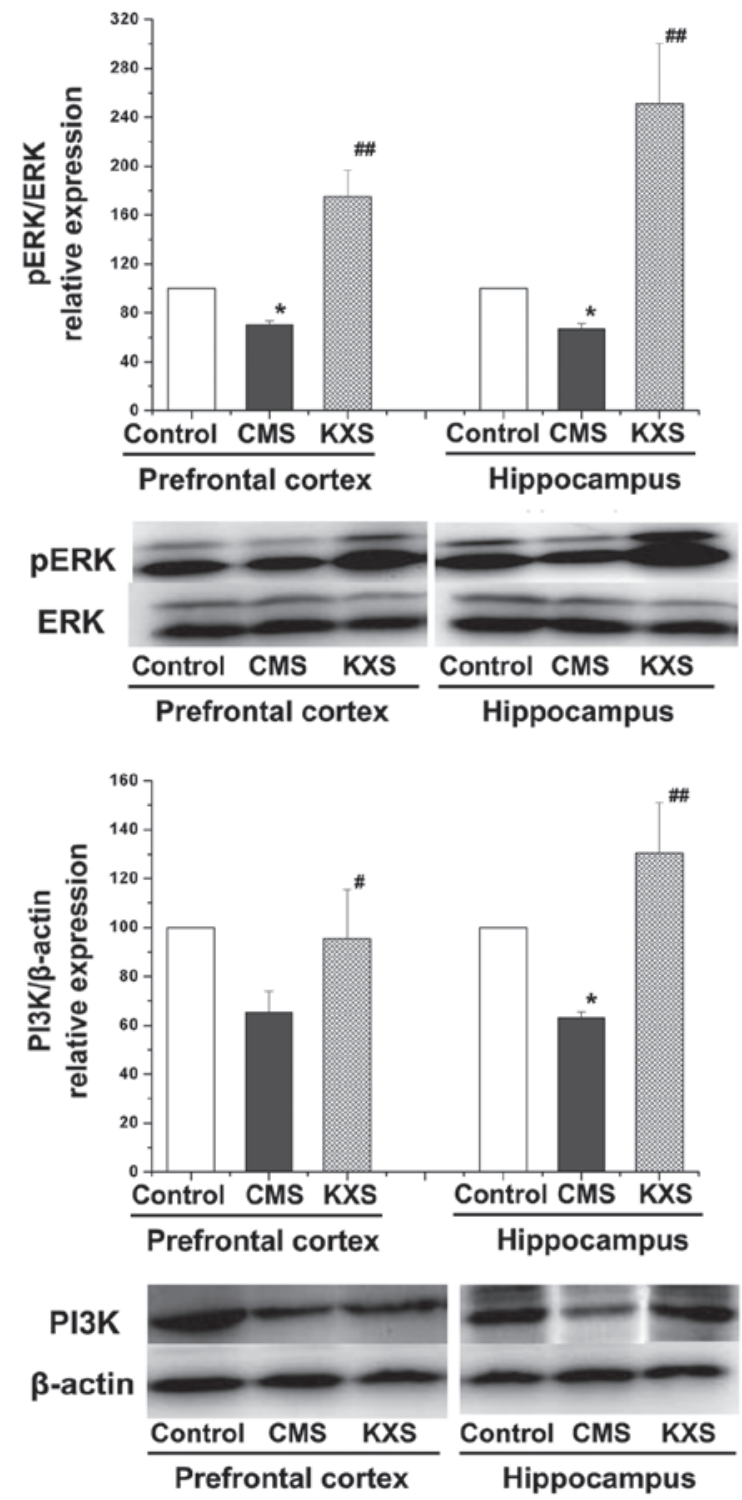

Figure 2. Effects of KXS on the TrkB/BDNF/ERK and TrkB/BDNF/PI3K signaling pathway in CMS rats. KXS ( $370 \mathrm{mg} / \mathrm{kg}$ ) was administered daily for three weeks. Data are presented as the mean \pm standard deviation, ${ }^{*} \mathrm{P}<0.05$ vs. the control group; ${ }^{\# \#} \mathrm{P}<0.01$ and ${ }^{\#} \mathrm{P}<0.05$ vs. the CMS group. CMS, chronic unpredictable mild stress; KXS, Kai-Xin-San; BDNF, brain-derived neurotrophic factor; Akt, protein kinase B; p, phospho; ERK, extracellular signal-regulated kinase; TrkB, tyrosine receptor kinase B; GSK3 $\beta$, glycogen synthase kinase $3 \beta$; PI3K, phosphatidylinositol-3-kinase.

Protein expression levels of pERK/ERK, PI3K, Akt and $p G S K 3 \beta / G S K 3 \beta$. Fig. 2 demonstrates that the protein expression levels of pERK/ERK $(\mathrm{P}<0.01)$, $\mathrm{PI} 3 \mathrm{~K}$ (hippocampus, $\mathrm{P}<0.01$; prefrontal cortex, $\mathrm{P}<0.05$ ), and Akt (hippocampus, $\mathrm{P}<0.01$; prefrontal cortex, $\mathrm{P}<0.05)$ in the hippocampus and prefrontal cortex, and pGSK3 $\beta / G S K 3 \beta(\mathrm{P}<0.05)$ in the hippocampus of the KXS group were significantly elevated, compared with the CMS group. The results suggest that the regulation effect of $\mathrm{KXS}$ on BDNF and pCREB might be related to its activation on the upstream signaling pathway of CREB.

Cell viability and BDNF expression. Fig. 3 shows MAP-2 immunostaining of neuronal cells. The morphous and purity of the neuronal cells was suitable for the experiment. In cells treated with KXS, cell viability was significantly increased, as compared with the control cells $(\mathrm{P}<0.01)$. The level of BDNF release increased in a time-dependent manner, peaking at $6 \mathrm{~h}$ post-treatment (Fig. 4). KXS could increase the nerve cell viability which might be involved in the increasing level of BDNF.

Protein expression levels of TrkB/ERK in neuronal cells. Fig. 5 indicates that the protein expression levels of BDNF, pERK and pTrkB in the KXS group were significantly higher than that of the control group $(\mathrm{P}<0.01)$. Such an increase was not observed when cells were treated with $\mathrm{K} 252 \mathrm{a}$, which is an inhibitor of TrkB. The effects of KXS on the pathway components in cells coincided with those of animal experiments.

\section{Discussion}

In CMS rats treated with $\mathrm{KXS}$, increased protein expression levels of pCREB, BDNF and TrkB were detected in the hippocampus and prefrontal cortex. Consistently, in primary 

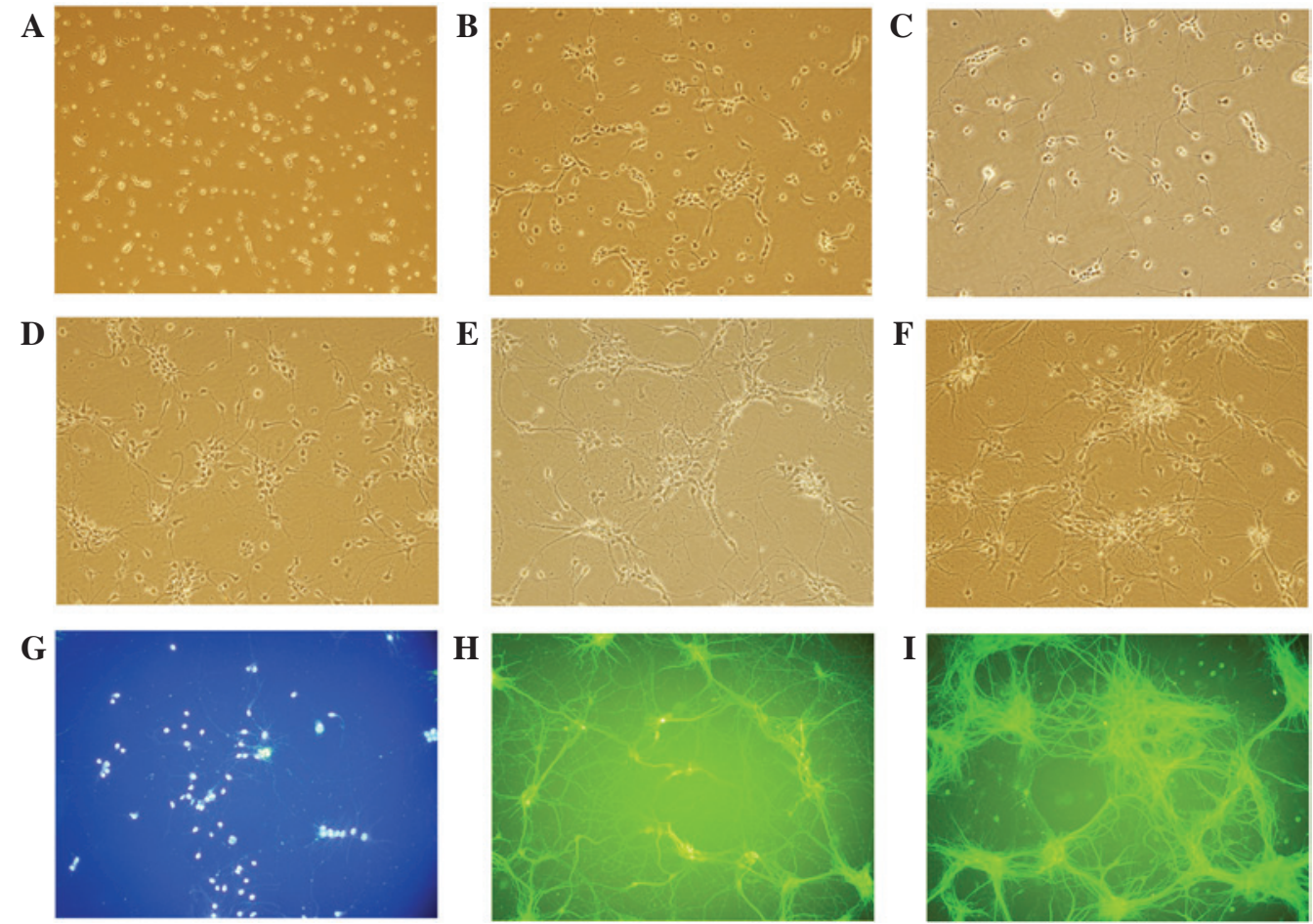

Figure 3. Morphological observation and identification of primary hippocampal neurons (magnification, x200) at (A) 4 h, (B) 24 h, (C) 2 days, (D) 3 days, (E) 5 days, (F) 7 days. Primary hippocampal neurons incubated with (G) DIPA and MAP-2 at (H) day 5 and (I) day 7.

A

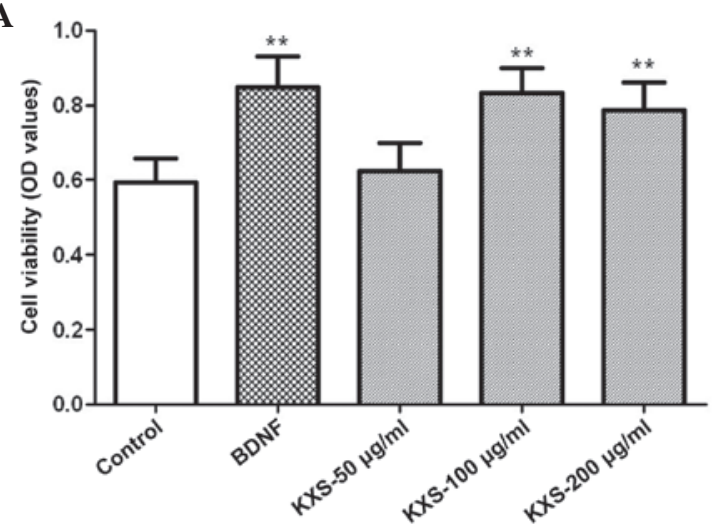

B

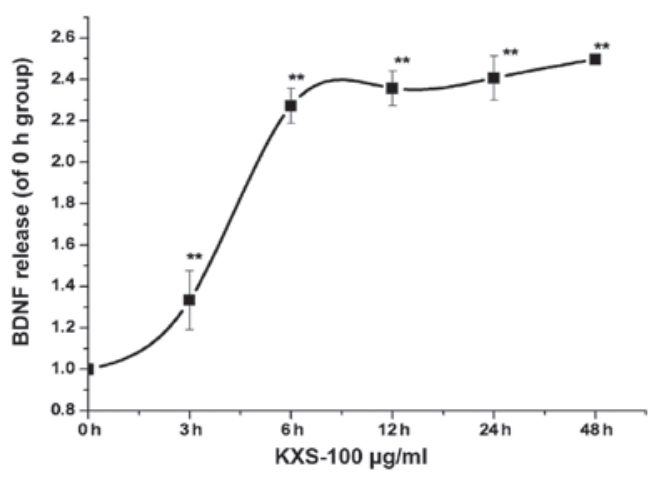

Figure 4. Effect of KXS on cell survial and BDNF release from primary hippocampal neurons. (A) For cell viability assay, cells were treated with $25 \mathrm{pg} / \mathrm{l}$ BDNF or 50,100 and $200 \mu \mathrm{g} / \mathrm{ml}$ of KXS for $48 \mathrm{~h}$. ${ }^{* *} \mathrm{P}<0.01$ vs. the control group. (B) In the BDNF release test, cultured cells were treated with $100 \mu \mathrm{g} / \mathrm{ml} \mathrm{KXS} \mathrm{for} 0,3$, $6,12,24$ and $48 \mathrm{~h} .{ }^{* *} \mathrm{P}<0.01$ vs. the $0 \mathrm{~h}$ group. Data are presented as the mean \pm standard deviation. KXS, Kai-Xin-San; BDNF, brain-derived neurotrophic factor; OD, optical density.

hippocampal neurons, an increased level of upstream components, including ERK, pERK, PI3K, Akt and GSK3 $\beta$, was observed when cells were treated with KXS. These results suggest that pCREB and upstream components may be associated with the antidepressive effect of KXS.

Depression is a complex mental disorder involving multiple factors. Impaired release of BDNF, which is a small protein utilized by the brain for development and nerve cell growth regulation and function (25), has been demonstrated to have a key role in major depression (26). The present finding of increased BDNF levels in CMS rats treated with KXS is consistent with previous reports (22). In previous animal and human studies, circulating levels of BDNF were very low and could be increased with treatment of antidepressants (27-30), which may account for its efficacy in depressive behaviors $(31,32)$.

It has been demonstrated that ERK and PI3K pathway are the two most important pathways altered through the BDNF mediated-TrkB activation, resulting in neuroprotection effect (33). ERKs are able to activate and transfer signals to the nucleus quickly, in order to induce CREB phosphorylation and activate the nuclear transcription factor, CREB. Enhanced CREB ultimately results in increased expression of BDNF (34). These data suggest a positive feedback loop between BDNF and CREB, which may be associated with nerve cell nutrition and nerve injury repair (35). In this study, increased pERK was observed in CMS rats treated with KXS; however, such an increase was not observed when primary 

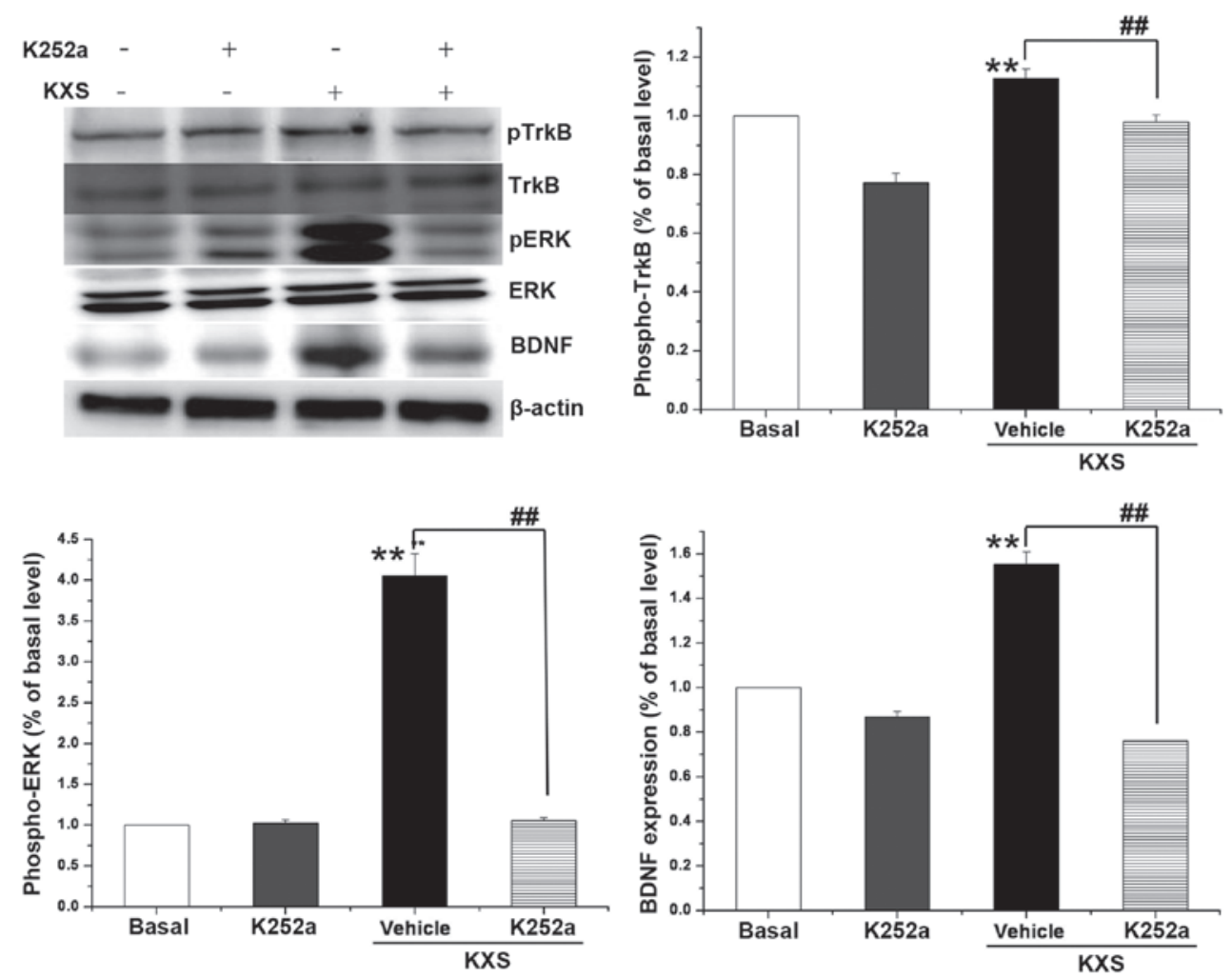

Figure 5. KXS rapidily activates the TrkB/ERK signaling pathway. Effects of K252a on the KXS ( $100 \mu \mathrm{g} / \mathrm{ml}$ for $48 \mathrm{~h})$-induced TrkB and ERK phosphorylation or BDNF expression were evaluated by western blot. Data are presented as the mean \pm standard deviation ${ }^{* *} \mathrm{P}<0.01$ vs. the basal group, ${ }^{\# \#} \mathrm{P}<0.01$ vs. the KXS alone. KXS, Kai-Xin-San; BDNF, brain-derived neurotrophic factor; ERK, extracellular signal-regulated kinase; TrkB, tyrosine receptor kinase B.

neurons cells were treated with $\mathrm{K} 252 \mathrm{a}$, which is an inhibitor of TrkB. This suggests that the KXS-induced increase of BNDF may be mediated by the TrkB-dependent ERK pathway. Activated CREB may further regulate the transcription of genes involved in neuronal cell survival.

GSK-3 $\beta$ is an essential pro-apoptotic factor for neuronal the apoptosis cascade and PI3K-mediated neuronal survival pathway (36). In its active form, GSK-3 $\beta$ is able to enhance apoptosis; however, once inactivated by phorphorylation, it cannot trigger apoptotic pathways (37). In the present study, inactivated GSK-3 $\beta$ by phosphorylation and an PI3K/AKT-mediated increase in BDNF was observed when rats were treated with $\mathrm{KXS}$. This finding is consistent with a previous report, demonstrating that BDNF may provide neuroprotection via the activation of the PI3K/Akt pathway (38).

In conclusion, increased protein expression levels of pCREB, BDNF and TrkB were detected in the hippocampus and prefrontal cortex. Consistently, in primary hippocampal neurons, increased levels of upstream components, including ERK, pERK, PI3K, Akt, and GSK3 $\beta$, were observed when cells were treated with KXS. These results suggest that pCREB and its upstream components may be associated with the antidepressive effect of KXS. Subsequent studies in other cells and animal models are required to confirm the present findings and conclusions.

\section{Acknowledgments}

This work was supported by grants from the National Natural Science Foundation (grant nos. 81302909 and 81173430).

\section{References}

1. Voleti B and Duman RS: The roles of neurotrophic factor and Wnt signaling in depression. Clin Pharmacol Ther 91: 333-338, 2012.

2. Sen S, Duman R and Sanacora G: Serum brain-derived neurotrophic factor, depression and antidepressant medications: Meta-analyses and implications. Biol Psychiatry 64: 527-532, 2008.

3. Karege F, Vaudan G, Schwald M, Perroud N and La Harpe R: Neurotrophin levels in postmortem brains of suicide victims and the effects of antemortem diagnosis and psychotropic drugs. Brain Res Mol Brain Res 136: 29-37, 2005.

4. Hsiung SC, Adlersberg M, Arango V, Mann JJ, Tamir H and Liu KP: Attenuated 5-HT1A receptor signaling in brains of suicide victims: Involvement of adenylyl cyclase, phosphatidylinositol 3-kinase, Akt and mitogen-activated protein kinase J Neurochem 87: 182-194, 2003.

5. Jernigan CS, Goswami DB, Austin MC, Iyo AH, Chandran A, Stockmeier CA and Karolewicz B: The mTOR signaling pathway in the prefrontal cortex is compromised in major depressive disorder. Prog Neuropsychopharmacol Biol Psychiatry 35: 1774-1779, 2011.

6. Dwivedi Y, Rizavi HS, Zhang H, Roberts RC, Conley RR and Pandey GN: Modulation in activation and expression of phosphatase and tensin homolog on chromosome ten, Akt1 and 3-phosphoinositide-dependent kinase 1: Further evidence demonstrating altered phosphoinositide 3-kinase signaling in postmortem brain of suicide subjects. Biol Psychiatry 67: 1017-1025, 2010

7. Molteni R, Calabrese F, Racagni G, Fumagalli F and Riva MA: Antipsychotic drug actions on gene modulation and signaling mechanisms. Pharmacol Ther 124: 74-85, 2009.

8. Fishback JA, Robson MJ, Xu YT and Matsumoto RR: Sigma receptors: Potential targets for a new class of antidepressant drug. Pharmacol Ther 127: 271-282, 2010.

9. Blendy JA: The role of CREB in depression and antidepressant treatment. Biol Psychiatry 59: 1144-1150, 2006.

10. Milnerwood AJ and Raymond LA: Early synaptic pathophysiology in neurodegeneration: Insights from Huntington's disease. Trends Neurosci 33: 513-523, 2010. 
11. Zhu Y, Duan X, Huang F, Cheng X, Zhang L, Liu P, Shulan S, Duan JA, Dong TT and Tsim KW: Kai-Xin-San, a traditional Chinese medicine formula, induces neuronal differentiation of cultured PC12 Cells: Modulating neurotransmitter regulation enzymes and potentiating NGF inducing neurite outgrowth. J Ethnopharmacol 193: 272-282, 2016.

12. Qiong W, Yong-Liang Z, Ying-Hui L, Shan-Guang C, Jiang-Hui G, Yi-Xi C, Ning J and Xin-Min L: The memory enhancement effect of Kai Xin San on cognitive deficit induced by simulated weightlessness in rats. J Ethnopharmacol 187: 9-16, 2016.

13. Zhu KY, Xu SL, Choi RCY, Yan AL, Dong TTX and Tsim KWK: Kai-Xin-San, a Chinese Herbal Decoction Containing Ginseng Radix et Rhizoma, Polygalae Radix, Acori Tatarinowi Rhizoma, and Poria, Stimulates the Expression and Secretion of Neurotrophic Factors in Cultured Astrocytes. Evid Based Complement Alternat Med 731385, 2013.

14. Dang H, Sun L, Liu X, Peng B, Wang Q, Jia W, Chen Y, Pan A and Xiao P: Preventive action of Kai Xin San aqueous extract on depressive-like symptoms and cognition deficit induced by chronic mild stress. Exp Biol Med (Maywood) 234: 785-793, 2009.

15. Hu Y, Liu P, Guo DH, Rahman K, Wang DX, Chen ML and Xie TT: Behavioral and biochemical effects of Kaixin-San, a traditional Chinese medicinal empirical formula. Drug Develop Res 69: 267-271, 2008.

16. Zhou XJ, Liu M, Yan JJ, Cao Y and Liu P: Antidepressant-like effect of the extracted of Kai Xin San, a traditional Chinese herbal prescription, is explained by modulation of the central monoaminergic neurotransmitter system in mouse. J Ethnopharmacol 139 : 422-428, 2012.

17. Dong XZ, Li ZL, Zheng XL, Mu LH, Zhang G and Liu P: A representative prescription for emotional disease Ding-Zhi-Xiao-Wan restores 5-HT system deficit through interfering the synthesis and transshipment in chronic mild stress-induced depressive rats. J Ethnopharmacol 150: 1053-1061, 2013.

18. Cao Y, Hu Y, Liu P, Zhao HX, Zhou XJ and Wei YM: Effects of a Chinese traditional formula Kai Xin San (KXS) on chronic fatigue syndrome mice induced by forced wheel running. J Ethnopharmacol 139: 19-25, 2012.

19. National Pharmacopoeia Committee. Pharmacopoeia of People's Republic of China [M]. Part 1. Beijing: Chemical Industry Press, 2010: 7-437.

20. Mu LH, Huang ZX, Liu P, Hu Y and Gao Y: Acute and subchronic oral toicity assessment of the herbal formula Kai-Xin-San. J Ethnopharmacol 138: 351-357, 2011.

21. Hu Y, Cao Y, Liu M, Liu P, Cui H and Dai-Hong G: Behavioral and biochemical effects of a formulation of the traditional Chinese medicine, Kai-Xin-San, in fatigued rats. Exp Ther Med 6: 973-976, 2013.

22. Hu Y, Liu M, Liu P, Guo DH, Wei RB and Rahman K: Possible mechanism of the antidepressant effect of 3,6'-disinapoyl sucrose from polygala tenuifolia willd. J Pharm Pharmacol 63: 869-874, 2011.

23. Liu D, Zhang H, Gu W, Liu Y and Zhang M: Neuroprotective effects of ginsenoside Rb1 on high glucose-induced neurotoxicity in primary cultured rat hippocampal neurons. PLoS One 8: e79399, 2013.

24. He XL, Zhang P, Dong XZ, Yang MH, Chen SL and Bi MG: JR6, a new compound isolated from Justicia procumbens, induces apoptosis in human bladder cancer EJ cells through caspase-dependent pathway. J Ethnopharmacol 144: 284-292, 2012.
25. Hu Y, Zhou XJ, Liu P, Dong XZ, Mu LH, Chen YB, Liu MY and Yu BY: Anti-depressant and neuroprotective effect of the Chinese herb KaiXinSan against lentiviral shRNA Knockdown brain-derived neurotrophic factor-induced injury in vitro and in vivo. Neuropsychobiology 69: 129-139, 2014.

26. Yu $\mathrm{H}$ and $\mathrm{Chen} \mathrm{ZY}$ : The role of BDNF in depression on the basis of its location in the neural circuitry. Acta Pharmacol Sin 32: 3-11, 2011.

27. Sen S, Duman R and Sanacora G: Serum brain-derived neurotrophic factor, depression and antidepressant medications: Meta-analyses and implications. Biol Psychiatry 64: 527-532, 2008.

28. Bernard R, Kerman IA, Thompson RC, Jones EG, Bunney WE, Barchas JD, Schatzberg AF, Myers RM, Akil H and Watson SJ: Altered expression of glutamate signaling, growth factor and glia genes in the locus coeruleus of patients with major depression. Mol Psychiatry 16:634-646, 2011.

29. Kozicz T, Tilburg-Ouwens D, Faludi G, Palkovits M and Roubos E: Gender-related urocortin 1 and brain-derived neurotrophic factor expression in the adult human midbrain of suicide victims with major depression. Neuroscience 152: 1015-1023, 2008.

30. Altar CA, Whitehead RE, Chen R, Wörtwein G and Madsen TM: Effects of electroconvulsive seizures and antidepressant drugs on brain-derived neurotrophic factor protein in rat brain. Biol Psychiatry 54: 703-709, 2003

31. Shirayama Y, Chen AC, Nakagawa S, Russell DS and Duman RS: Brain-derived neurotrophic factor produces antidepressant effects in behavioral models of depression. J Neurosci 22: 3251-3261, 2002.

32. Deltheil T, Tanaka K, Reperant C, Hen R, David DJ and Gardier AM: Synergistic neurochemical and behavioural effects of acute intrahippocampal injection of brain-derived neurotrophic factor and antidepressants in adult mice. Int J Neuropsychopharmacol 12: 905-915, 2009.

33. Jain V, Baitharu I, Prasad D and Ilavazhagan G: Enriched Environment prevents hypobaric hypoxia induced memory impairment and neurodegeneration: Role of BDNF/PI3K/GSK3 $\beta$ pathway coupled with CREB activation. PLoS One 8: e62235, 2013.

34. Yan X, Liu J, Ye Z, Huang J, He F, Xiao W, Hu X and Luo Z: CaMKII-Mediated CREB Phosphorylation Is Involved in $\mathrm{Ca}^{2+}$-Induced BDNF mRNA Transcription and Neurite Outgrowth Promoted by Electrical Stimulation. PLoS One 11: $\mathrm{e} 0162784,2016$.

35. Fišar Z and Hroudová J: Intracellular signalling pathways and mood disorders. Folia Biol (Praha) 56: 135-148, 2010.

36. Dong XZ, Huang CL, Yu BY, Hu Y, Mu LH and Liu P: Effect of Tenuifoliside a isolated from polygala tenuifolia on the ERK and PI3K pathways in C6 glioma cells. Phytomedicine 21: 1178-1188, 2014.

37. Zhang JS, Herreros-Villanueva M, Koenig A, Deng Z, de Narvajas AA, Gomez TS, Meng X, Bujanda L, Ellenrieder V, Li XK, Kaufmann SH and Billadeau DD: Differential activity of GSK-3 isoforms regulates NF- $\kappa$ B and TRAIL- or TNF $\alpha$ induced apoptosis in pancreatic cancer cells. Cell Death Dis 5: e1142, 2004.

38. Zhang L, Zhao H, Zhang X, Chen L, Zhao X, Bai X and Zhang J: Nobiletin protects against cerebral ischemia via activating the p-Akt, p-CREB, BDNF and Bcl-2 pathway and ameliorating BBB permeability in rat. Brain Res Bull 96: 45-53, 2013. 